\title{
Germanica
}

\section{Passeurs et intermédiaires de Schnitzler en France : essai d'une typologie des agents du transfert}

Transmitters and intermediaries of Schnitzler in France: an essay in the typology of cultural middle-men

Vermittler der Werke A. Schnitzlers in Frankreich. Ansätze zu einer Typologie

\section{Karl Zieger}

\section{(2) OpenEdition}

Journals

Édition électronique

URL : http://journals.openedition.org/germanica/2116

DOI : 10.4000/germanica.2116

ISSN : 2107-0784

Éditeur

Université de Lille

\section{Édition imprimée}

Date de publication : 15 juin 2013

Pagination : 13-24

ISBN : 9782913857315

ISSN : 0984-2632

Référence électronique

Karl Zieger, «Passeurs et intermédiaires de Schnitzler en France : essai d'une typologie des agents du transfert », Germanica [En ligne], 52 | 2013, mis en ligne le 20 juin 2013, consulté le 06 octobre 2020

URL : http://journals.openedition.org/germanica/2116; DOI : https://doi.org/10.4000/germanica.2116 


\title{
Passeurs et intermédiaires de Schnitzler en France:
}

\author{
essai d'une typologie des agents du transfert
}

\author{
Karl ZIEGER \\ Université Charles-de-Gaulle - Lille 3
}

Dans le cadre d'un projet visant la réalisation d'un « dictionnaire des agents du transfert culturel $»^{1}$, cet article s'intéresse aux traducteurs et «agents littéraires » comme éléments décisifs pour l'accueil d'un écrivain et de son œuvre à l'étranger et dans une langue étrangère. Dans cette perspective, l'accueil réservé à l'œuvre d'Arthur Schnitzler dans le champ littéraire français constitue un cas intéressant, voire paradigmatique $^{2}$.

Les conditions de travail et le statut des «passeurs et intermédiaires » ont été pendant longtemps négligés par la critique et c'est seulement depuis une dizaine d'années qu'ils font l'objet de la recherche universitaire. Parmi les pionniers d'une approche sociologique, maté-

1. - Le colloque sur « Nestroy, Schnitzler et leur relations avec la France » s'inscrit dans un projet plus vaste dont le but est l'établissement d'une «prosopographie » des personnes impliquées dans les échanges culturels entre la France et l'Autriche au XIXe et dans le premier tiers du XXe siècle. Les contributions à deux manifestations précédentes viennent d'être publiées en co-édition par innsbruck university press ( Germanistische Reihe ») et les PU de Valenciennes (Sigurd Paul Scheichl et Karl Zieger, sous la direction de : France-Autriche: leurs relations culturelles 1867-1938, Innsbruck, Valenciennes, 2012).

2. - Voir à ce propos Karl Zieger: Arthur Schnitzler et la France 1894-1938: enquête sur une réception, Villeneuve d'Ascq, Presses universitaires du Septentrion, 2012. (Les faits présentés dans cet article proviennent essentiellement de ce travail). 
rielle et bibliométrique de cette activité compte Blaise Wilfert-Portal qui a consacré à ce sujet sa thèse de doctorat intitulée Paris, la France et le reste. Importations littéraires et nationalisme culturel 1885-1930, soutenue en 2003 à Paris I et deux articles très éclairants ${ }^{3}$, ainsi que le chapitre «Traduction littéraire: une approche bibliométrique » dans le tout récent premier volume de l'Histoire des traductions en langue française portant sur le XIX ${ }^{\mathrm{e}}$ siècle ${ }^{4}$.

Wilfert propose pour ses « agents de la réception » le terme « d'importateurs littéraires » et désigne ainsi tous ceux qui ont une part de responsabilité dans la genèse d'une traduction, que ce soit en tant qu'éditeur, directeur de collection, agent littéraire, ainsi que ceux qu'il appelle « les pionniers qui ont indiqué que l'œuvre méritait d'être traduite, ceux qui enfin plaident la cause de cette traduction comme critiques ou comme historiens des littératures, construisant sa valeur dans l'espace récepteur $»^{5}$. Le terme d'importateur a, pour lui, l'avantage « d'écarter les mythologies rétrospectives et les représentations léguées par les intéressés eux-mêmes ». Et dans ses travaux, Wilfert montre, en effet, la situation souvent précaire des « passeurs » d'œuvres culturelles, dont certains font des traductions "à la chaîne ", alors que pour d'autres il s'agit d'un travail occasionnel.

En appliquant des critères de sélection assez stricts - « une traduction, un article, une préface ne peuvent suffire à faire d'un écrivain un importateur »-Wilfert a pu identifier, pour la période 1885-1914, « une centaine de noms pour lesquels l'importation sous ses diverses formes a tenu, à un moment au moins, un rôle essentiel dans leur position dans le champ intellectuel et dans leur identité sociale ${ }^{6}$. Dans son article sur les « importateurs », Blaise Wilfert ne cite cependant pas ces cent noms, mais procède par centre d'intérêt en évoquant successivement le travail de traducteur, les modes éditoriales, le rôle des revues dans l'importation littéraire et les stratégies de l'importation, activité dans laquelle les femmes, les jeunes et les diplomates joueraient un rôle important.

Il faut aussi rappeler que la traduction n'était pas, à la fin du XIXe/ au début du $\mathrm{XX}^{\mathrm{e}}$ siècle, une activité bien protégée, qu'elle était consi-

3. - « Cosmopolis et L'Homme invisible. Les importateurs de littérature étrangère en France, 1885-1914 », in Actes de la recherche en sciences sociales, $\mathrm{n}^{\circ} 144$, septembre 2002, p. 33-46 et « Des bâtisseurs de frontières. Traduction et nationalisme culturel en France, 1880-1930 », in Christine Lombez et Rotraud von Kulessa (éds.) : De la traduction et des transferts culturels, Paris, L'Harmattan, 2007, p. 231-253.

4. - Yves Chevrel, Lieven d'Hulst et Christine Lombez (sous la direction de): Histoire des traductions en langue française. XIXe siècle : 1815-1914, Lagrasse, Verdier, 2012. Le chapitre rédigé par B. Wilfert-Portal s'y trouve p. 255-344.

5. - Blaise Wilfert, "Cosmopolis et L'Homme invisible. Les importateurs de littérature étrangère en France »,op.cit., p. 34.

6. - Ibid. 
dérée comme une activité « mineure » qui ne permettait pas, à quelques exceptions près, d'accéder à une place valorisée dans le champ littéraire, d'obtenir le statut d'auteur ${ }^{7}$. La compétence linguistique des traducteurs était considérée comme secondaire; ce qui comptait, c'était « la mise en français » des textes étrangers.

En effet, dans son article sur « Les bâtisseurs de frontières », Wilfert démolit l'image des années 1880/1930 comme un "âge d'or » de la traduction et du cosmopolitisme. Il estime que l'on sait, en fait, très peu de choses sur les nombreux traducteurs qui ont œuvré à l'époque en France et que le Catalogue général de la librairie française est, à ce propos, très lacunaire. Seuls quelques noms «emblématiques », comme celui d'Elie Halpérine-Kaminski pour ses traductions du russe et celui d'Alexandre Vialatte pour ses traductions de Kafka, sortent de l'anonymat... ou ceux d'écrivains reconnus qui ont signé des traductions, comme c'est le cas d'André Gide. C'est justement à propos de Gide que Wilfert montre que l'auteur des Faux-Monnayeurs, quand il a commencé, dans les années 1910, à faire des traductions de l'anglais (Tagore, Whitman, Conrad, Meredith et même Hamlet) était encore loin d'avoir toutes les compétences requises dans la langue de Shakespeare ${ }^{8}$ et avait surtout pour tâche de «domestiquer » le texte étranger, de le «naturaliser français », la fidélité à l'œuvre n'étant pas considérée comme l'essentiel. Ce qui explique aussi que bon nombre de traducteurs se sont attaché les services d'un « native speaker » ou d'un traducteur assermenté qui leur fournissait une première traduction « littérale » de l'œuvre étrangère, traduction qu'ils mettaient ensuite « en bon français ». Le constat que fait Blaise Wilfert est, en tout cas, clair: pour lui, la traduction française au temps de la Troisième République se caractérise par une « domestication consciente et théorisée $»^{9}$ du texte étranger.

Les phénomènes constatés par Wilfert se trouvent, sans surprise, aussi dans la réception des œuvres de Schnitzler en France. Sans entrer dans les discussions sur le degré de «domestication » et d'« adéquation » qu'atteignent les traductions, on peut esquisser, en schématisant, une typologie des agents littéraires et traducteurs qui ont œuvré pour rendre les textes de Schnitzler accessibles en français.

Une première catégorie d'intermédiaires ne sera évoquée que brièvement: ceux qui ont pu établir les premiers contacts entre l'écrivain et ses traducteurs. Il s'agit, dans le cas de Schnitzler, de connaissances du milieu intellectuel viennois qui, pour des raisons professionnelles ou person-

7. - Ibid., p. 36 .

8. - Voir Blaise Wilfert-Portal: « Des bâtisseurs de frontières. Traduction et nationalisme culturel en France », op. cit., notamment p. 242-246.

9. - Ibid., p. 252. 
nelles avaient des liens avec la France ou y résidaient: ce sont, essentiellement, Paul Goldmann (1865-1935), ami de longue date de Schnitzler qui a séjourné à Paris dans les années 1890 comme correspondant de la Frankfurter Zeitung et établi le contact avec Henri Albert, l'auteur des premiers articles sur Schnitzler dans le Mercure de France, et Jean Thorel, auteur de la première traduction de Liebelei [Amourette] ; Berta Zuckerkandl (1864-1945), la Hofrätin [« Conseillère aulique »], comme on l'a appelée dans les milieux artistiques et intellectuels viennois, qui a animé dans les années 1920/30 les échanges culturels franco-autrichiens en œuvrant pour la traduction d'écrivains français en Autriche et d'écrivains autrichiens en France, et, dans une moindre mesure, Paul Zifferer (1879-1928) qui a été, dans les années 1920 également, publizistischer Berater [" conseiller en communication »] à l'ambassade d'Autriche à Paris ${ }^{10}$. Le rôle exact de ces «premiers intermédiaires » mériterait un travail à part ${ }^{11}$.

Ici, nous nous limiterons à évoquer le travail de deux traducteurs... et d'un agent littéraire. Parmi les traducteurs, on peut distinguer les professionnels de la traduction et les « amateurs».

On entendra par «professionnels »12 ceux qui, même s'ils peuvent avoir un autre métier par ailleurs (souvent d'enseignant ou de journaliste), font de la traduction un « gagne-pain » ou, en tout cas, un revenu supplémentaire et traduisent, parfois, de manière quasi-industrielle : c'est notamment le cas de Maurice Rémon (1861-1945) dont Pierre Debofle a dressé l'inventaire des très nombreuses traductions de l'allemand et de l'anglais ${ }^{13}$. C'est aussi le cas de Jean Thorel, d'Alzir Hella (qui se fera un nom comme traducteur de Stefan Zweig), voire de Maurice Vaucaire; ce dernier, étant lui-même auteur dramatique, correspond cependant plutôt à la catégorie des écrivains-traducteurs. Ces traducteurs apparaissent souvent en binôme, travaillant en l'occurrence assez régulièrement avec l'aide de native speakers ou d'interprètes: Noémi Valentin et Wilhelm Bauer pour Rémon, Olivier Bournac pour Alzir Hella.

10. - Voir dans ce volume l'article de Jacques Le Rider, p. 25.

11. - À propos du rôle de Berta Zuckerkandl dans la vie intellectuelle viennoise, voir ses mémoires Ich erlebte fünfzig Jahre Weltgeschichte, Stockholm, 1939, traduit en français par Maurice Rémon: Souvenirs d'un monde disparu : Autriche 1878-1938, Paris, Calmann-Lévy, 1939, et une biographie récente due à Michael Schutte, Berta Zuckerkandl. Salonière, Journalistin, Geheimdiplomatin, Zurich, Atrium-Verlag, 2006.

12. - Dans le chapitre II « Traducteurs » de l'Histoire des traductions en langue française (op. cit., p. 149-187), Susan Pickford retrace la « lente et incertaine marche [des traducteurs] vers la professionnalisation » (p. 149) avec une typologie bien plus fine, mais essentiellement basée sur le XIX ${ }^{\mathrm{e}}$ siècle.

13. - Voir Pierre Debofle, « un traducteur parisien de la première moitié du XXe siècle : Maurice Rémon (1861-1945) à travers son journal personnel », in Bulletin de la Société de l'histoire de Paris et de l'Île de France, 108e année, 1981, p. 151-216. 
Les relations de l'écrivain avec ce type de traducteur restent, en dehors de la discussion sur le choix des œuvres à traduire, souvent plutôt dans le domaine des « affaires commerciales », elles portent par exemple sur le pourcentage des droits accordé à l'un et à l'autre; leur correspondance contient plutôt rarement des réflexions littéraires sur la traduction; néanmoins, elle peut nous renseigner sur la situation et les pratiques de l'édition « étrangère » en France à cette époque. Ainsi, la correspondance de Schnitzler avec Maurice Rémon révèle les difficultés et les obstacles que les intermédiaires ont à surmonter pour placer une œuvre étrangère dans un théâtre comme l'Odéon dirigé, à cette époque, par André Antoine: malgré des promesses répétées (il semble même qu'une déclaration officielle aurait été déposée auprès de la SACD), Antoine, qui avait déjà mis en scène, au Théâtre Antoine, deux pièces en un acte de Schnitzler, Le Perroquet vert et La Compagne, ne montera aucune des saynètes du cycle Lebendige Stunden. Parmi les explications et les excuses qu'il donne pour les reports répétés, celle citées par Rémon dans sa lettre du 30 octobre 190714 mérite quelque attention. Selon Rémon, Antoine lui aurait déclaré :

Je n'ai pas pu monter de suite La Femme au poignard parce que c'est d'un auteur étranger, et dans un théâtre subventionné comme l'Odéon je ne suis pas tout à fait libre, les jeunes poètes français ne manqueraient pas de crier si je rouvrais au début de la saison avec une pièce étrangère. Puis j'ai senti que la pièce est trop artiste et d'un genre trop spécial pour aller avec la comédie de Bisson, œuvre très légère, presque Vaudeville. Je l'ai donc enlevée, mais vous pouvez annoncer à $\mathrm{M}$. Schnitzler que je vais la mettre en répétition de suite, dès le 8 courant, la 1ères [sic] des Bisson ayant lieu le 7, avec Mme Van Doren, Vargas dans le jeune homme et Garnier sans doute dans le vieux Remigio.

De même, la réaction de Rémon après sa lecture de Das weite Land éclaire l'horizon d'attente du public (voire des directeurs de théâtre) en France dans les années 1910 :

J'ai enfin pu achever la lecture de votre pièce; je la trouve très jolie et le dialogue en a un charme et une vivacité incomparables. Malgré cela je ne pense pas qu'il y ait lieu de la traduire et que nous ayons quelque chance de la faire jouer à Paris. Les personnages sont un peu trop spéciaux, trop exceptionnels pour être compris et d'autre part l'action et l'intérêt se dispersent trop pour le goût de notre public, de plus en plus exigent. Croyez que pour ma part je le regrette vivement, mais

14. - Les lettres de Rémon à Schnitzler sont conservées à l'Université de Cambridge, celles de Schnitzler à Rémon à la Handschriftenabteilung du Deutsches Literaturarchiv, Marbach/Neckar. 
mon collaborateur Bauer à qui j'en ai parlé est exactement du même avis que moi.

On ne sait pas, s'il faut prendre ces propos comme des compliments pour la pièce ou pour une critique par rapport à son inadéquation au goût français. Toujours est-il que Schnitzler a regretté toute sa vie que les hommes (et femmes) de théâtre français aient négligé ses « grandes pièces $»$ au profit des pièces en un acte et de Reigen ${ }^{15}$.

La correspondance entre Rémon et Schnitzler évoque aussi un problème assez symptomatique des habitudes éditoriales, à savoir la pratique de « gonfler » le volume d'un livre en y ajoutant des textes non contenus dans l'original. Ce problème se pose pour la publication de la traduction des sept saynètes du cycle Anatole :

P. V. Stock trouvant l'ensemble un peu «maigre » pour un livre qui doit se vendre au prix de trois francs cinquante, il décide d'y ajouter Die Gefährtin [La Compagne] dans la traduction déjà existante de Maurice Vaucaire. Dans un premier temps, Schnitzler s’insurge contre cette idée :

wozu ich aber unbedingt mein Einverständnis verweigere, ist die Aufnahme der „Gefährtin“ in den Anatol-Band.

Anatol ist ein vollkommen in sich geschlossenes Buch, besteht aus sieben Einaktern, in jedem Land, in dem es bisher erschienen ist, reichte das für den Band aus, und ich kenne französische Bücher zu 3 frcs. 50, die viel weniger enthalten,

écrit-il le 23 décembre 1912 à Rémon. Du point de vue artistique (littéraire) un tel ajout serait, pour lui, inacceptable. Dans une longue lettre datée du 27 décembre, Rémon essaie alors de le convaincre de la nécessité d'accepter la solution proposée par Stock. Le motif principal de l'éditeur est, écrit-il, que « le volume serait trop mince, trop creux et aurait trop de blancs ». Il cite comme exemple la traduction de La Ronde (dont Rémon a signé, avec Wilhelm Bauer, la traduction parue chez Stock en cette même année 1912) qui se vendrait mal, parce que le volume ne serait pas assez épais. Ensuite, Rémon fait miroiter à Schnitzler un autre avantage que représenterait l'ajout de La Compagne aux saynètes d'Anatole: «par son caractère plus sérieux, plus fort, elle préparera le lecteur aux autres volumes, théâtre et nouvelles, que nous voulons faire paraître ensuite et qui feront connaître aux lecteurs français une autre face, plus élevée encore, de votre talent ».

Il rassure le dramaturge aussi sur la présentation du volume : $L a$ Compagne sera bien à part, à la fin du volume qui ne portera, sur la

15. - Voir à ce propos le (sous-)chapitre «Reigen contre Weites Land», in K. Zieger: Arthur Schnitzler et la France...,op. cit., p. 176-197. 
couverture, que le seul titre Anatole. Schnitzler finit par se ranger aux arguments de Stock et de Rémon : le 31 décembre 1912, il donne son accord à une édition du cycle Anatole complété par La Compagne - à regret, bien entendu, car il répète que, pour lui, la solution la plus simple aurait été de vendre un volume contenant uniquement les saynètes d'Anatole un peu moins cher:

[...] finde aber, dass man einen besseren Ausweg hätte finden können. Der einfachste schiene mir ja noch immer der, dass man das Buch einfach billiger verkaufte; der Einheitspreis von 3 frcs. 50 ist mir immer ganz unverständlich gewesen. „Anatol“ als ein dünnes Bändchen (und er ist gar nicht so dünn) zu etwa 2 frcs. Würde sich natürlich heute, wo mein Name in Frankreich noch so wenig bekannt ist, viel besser verkaufen.

À l'opposé (du point de vue institutionnel) des traducteurs professionnels, on peut trouver, pour Schnitzler comme sans doute pour d'autres écrivains, des « amateurs » qui se mettent au service de l'auteur, des personnes souvent plus ou moins proches de lui et qui ont une approche de leur travail que l'on pourrait qualifier d'« émotionnelle».

C'est le cas de Clara Katharine Pollazcek (1875-1951), écrivaine et compagne attitrée de Schnitzler après son divorce d'avec Olga, qui fournit, probablement à sa demande, une première version de la traduction française de Fräulein Else, une traduction qui sera, avant publication, retravaillée par Germaine Delamain, l'épouse du co-gérant des éditions Stock; mais c'est surtout le cas de Suzanne Clauser (alias Dominique Auclères, 1898-1981), qui, au moment où elle se présente chez Schnitzler, à l'automne 1928, pour lui demander le droit de traduire ses œuvres en français, n'avait aucune place dans le champ littéraire français et pour seule compétence le fait d'être parfaitement bi-lingue. C'est sans doute essentiellement grâce aux relations professionnelles de son frère, un banquier installé à Paris, qu'elle réussira à publier ses premières traductions de nouvelles de Schnitzler dans l'hebdomadaire à grand tirage Gringoire (huit nouvelles rien qu'en 1929 et 1930). Par la suite, elle accomplira un travail considérable pour la connaissance de l'œuvre de l'écrivain viennois en France, même si on peut se demander dans quelle mesure ses traductions ont contribué à faire de Schnitzler un « écrivain français ». Elle est, aujourd'hui, effectivement critiquée pour ses traductions « ciblistes » qui frôlent parfois l'adaptation ${ }^{16}$. À

16. - Sur le travail de Suzanne Clauser voir Elsbeth Dangel : « Das Elend der Übersetzung. Bemerkungen zu Dominique Auclères Schnitzlerübersetzungen », in Modern Austrian Literature, vol. 17, n¹, 1984, p. 49-57; Renate Wagner: " "Unsere geschäftlich-literarischen Beziehungen". Arthur Schnitzler und Suzanne Clauser », Neue Zürcher Zeitung, 17 mars 1989, p. 41-42; Karl Zieger : «Schnitzler et la France : le 
son époque, ce n'était pas une rareté et on peut ici penser au constat de Jean-Louis Backès à propos des traductions françaises de Dostoïevski, à savoir que : « il semble aller de soi à la fin du XIX siècle qu'un traducteur doit plier l'original aux habitudes de la langue dans laquelle il traduit, et non pas sa propre langue aux particularités stylistiques de l'original $»^{17}$.

Dans le domaine des échanges culturels, les « agents littéraires », qui sont d'ailleurs souvent autoproclamés comme tels, méritent une attention particulière. Il s'agit de personnes ayant à la fois le goût de la littérature et de la création artistique ET des affaires et qui se proposent de servir d'intermédiaire entre écrivains, traducteurs, directeurs de théâtre et maisons d'édition.

Pour ce qui est des relations de Schnitzler avec la France, on retiendra ici, comme exemple, le cas d'un certain Stéphane Epstein qui a essayé d'assurer et d'assumer ce rôle entre 1902 et 1908, sans obtenir de résultats significatifs. L'adjectif indéfini « certain » se justifie ici par le fait que l'activité d'Epstein et sa biographie comportent une bonne part de mystères et de zones d'ombre : selon les différents éléments biographiques qui se trouvent notamment dans les notes, lettres et journaux de Schnitzler et dans des catalogues de bibliothèques, il serait né en 1866 en Basse-Autriche, publierait aussi sous le pseudonyme Stéphane Estienne et serait médecin, écrivain et traducteur. Il aurait fait des études de psychologie, de Sciences naturelles et un doctorat de philosophie et de médecine. Sa thèse soutenue à Berne en 1894 porte sur Die vier Rechnungsoperationen mit Besselschen Funktionen nebst einer geschichtlichen Einleitung. D'autres publications répertoriées sont : Naturwissenschaft und Cabbala, 1891; Bourget als Lyriker, 1893; Heinrich von Helmholtz, 1896; E. du Bois-Reymond, 1897; Hundert Jahre in Wort und Bild. Eine Kulturgeschichte des 19. Jahrhunderts, Berlin, 1899, Maupassant und der französische Roman der Gegenwart, 189918. Le 20 avril 1904 Schnitzler note dans son Tagebuch qu'il a travaillé comme assistant du «physiologue » Emil Heinrich Du BoisReymond (1818-1896).

travail d'intermédiaire de Suzanne Clauser », in Rolf Wintermeyer et K. Zieger (études réunies par), Les « Jeunes Viennois » ont pris de l'âge. Les œuvres tardives des auteurs du groupe «Jung Wien » et de leurs contemporains autrichiens, Valenciennes, Presses Universitaires de Valenciennes, 2004 (= Recherches Valenciennoises n 16), p. 139-150, ainsi que le sous-chapitre consacré à S. Clauser dans K. Zieger: Arthur Schnitzler et la France, op. cit., p. 75-90.

17. - Cité par Blaise Wilfert-Portal, « Des bâtisseurs de frontières... »,op.cit., notamment p. 251.

18. - Cf. Kürschners Deutscher Literaturkalender auf das Jahr 1904 hrsgg. von Dr. Heinrich Klenz, Leipzig, Göschen'sche Verlagshandlung. 
La biographie qu'il donne lui-même dans une lettre (inédite) à Schnitzler du 31 janvier $1904^{19}$ est curieusement plus énigmatique :

Que voulez-vous ? [en français dans le texte] Wenn man als russischer Jude mit einer Katholikin zur Frau in Deutschland erzogen wurde, um schliesslich als französischer Literat zu landen, da ist es nicht leicht, sein seelisches Gleichgewicht harmonisch aufrecht zu erhalten.

Dans le Tagebuch de Schnitzler on trouve des entrées sur Epstein qui montrent à la fois l'utilité de ce genre d'intermédiaire, mais aussi les réticences de l'écrivain. En voici quelques exemples:

28 décembre 1903 (rencontre avec $M^{\text {me }}$ Epstein, en visite à Vienne) : Bei Frau Epstein, der Gattin des Dramaturgen bei Antoine, über geschäftliche Dinge, event. Aufführung der letzten Masken und Literatur mit dem Kakadu u.a.

20 avril 1904 : Nm. Dr. Epstein, Paris, früher Assistent von Dubois [...]. Jetzt Dramaturg bei Antoine, dessen Vielgereistheit, Vielseitigkeit und Fleiss mir imponiert. Es ist doch recht wahrscheinlich dass ich mehr bin - und doch fühl ich mich gerade solchen Geschäftigen gegenüber oft als der Geringere.

21 avril 1904 : Vm. Besorgungen Notar (Vollmacht für Epstein).

8 mai 1905 : Zu Mittag Dr. Steph. Epstein (auch Liesl) da. Epstein recht schwadroneurig, aber leidlich amüsant, und durch seine Bethulichkeit wohl in unsern Angelegenheiten von Nutzen. - (Er ist mein bevollmächtigter Vertreter in Paris.).

13 novembre 1905 : Claire Tagger Nm. da, macht mir Mittheilungen über 'Reigen' den sie übersetzen will (zur Aufführung bei Antoine); Schwierigkeiten wegen Epstein, der die Autoris. noch hat und schwadronirt und lügt.

2 décembre 1905 : Frau Tagger bei mir mit Brief von Antoine, dass er die (Epstein) Übersetzung des Reigen nicht verwenden könne. (Frau T. will ihn übersetzen.)

3 février 1906 : Vorm. bei Dr. Epstein (Paris) Hotel Tegetthoff. Besorgungen.

11 février 1906 : Abd. Epstein und Soulié [= Maurice Soulié, Schriftsteller] (Paris) da; über die Autorenverhältnisse; S. : mais on vous a volé une petite fortune en Amérique.

9 avril 1907 : Bei Frau Rotenstern Tesi (wo Frl. Hermann und Mutter) die mir von Nizza erzählte; Association und Uneinigkeit ihres Manns mit Epstein in Paris (meinem « Vertreter » und Schwadroneur);

19. - La correspondance entre Schnitzler et Epstein se trouve à la Handschriftenabteilung du Deutsches Literaturarchiv, Marbach/Neckar. Sur Epstein, qui a aussi contribué à la traduction et la représentation en France d'Elektra de Hugo von Hofmannsthal, voir dans ce numéro l'article de Martine Sforzin, p. 37. 
Toujours est-il que, si Schnitzler admire l'activité débordante d'Epstein (voir la note du 20 avril 1904), il doit aussi se rendre compte que, malgré toutes ses promesses et toute son agitation, il n'arrive pas vraiment à «placer » ses œuvres en France. Car, malgré de nombreu $x$ projets évoqués, la seule « réalisation » d'Epstein est la traduction, en collaboration avec Émile Lutz, de Der Grüne Kakadu, mise en scène par André Antoine au Théâtre Antoine en novembre 1903, sous le titre $A u$ Perroquet vert, et Schnitzler finit par le considérer comme un «Schwadroneur», un « vantard».

Quant à la traduction du Kakadu, la correspondance entre Schnitzler et Epstein permet d'en suivre certaines étapes, par exemple quelques modifications que Lutz et Epstein proposent à Schnitzler. Elles concernent essentiellement des noms propres, que ce soit des noms de rue - le « Boulevard des Capucines », sur lequel est situé le cabaret de Prosper, prend, dans la traduction française, son nom du XVIII siècle, à savoir «Boulevard de la Madeleine » (lettre d'Epstein du 12 avril 1902) - ou des patronymes : «Grain » est rebaptisé «Legrain », parce que le nom monosyllabique sonnerait mal à l'oreille française ; "Henri Baston » serait un nom impensable pour un cabotin du XVIII ${ }^{\mathrm{e}}$ siècle, Lutz et Epstein préfèrent donc l'appeler « Didier Bâtonnard »; le nom de « Rollin » serait trop vite, trop facilement associé à celui du philosophe janséniste, pour qu'on puisse le donner à un auteur de farces, il sera donc remplacé par «Collin » ${ }^{20}$. Pour illustrer ses propos, Epstein demande à Schnitzler d'imaginer un instant, dans une pièce burlesque, un personnage qui s'appellerait «Immanuel Kant». Ces modifications seraient nécessaires, explique Epstein, car : " wir leben hier in einem Lande, wo der historische Sinn (fuer eigene Geschichte) so entwickelt ist, dass man Alles vermeiden muss, was der Presse Anlass zu Ausstellungen [sic] geben könnte » (lettre du 27 avril 1902).

Les lettres d'Epstein sont, en général, très longues, bavardes, verbeuses. En prenant quelques précautions, on peut néanmoins en tirer des renseignements sur des personnalités du milieu théâtral parisien de l'époque, sur la situation des dramaturges étrangers et sur le travail des traducteurs. À ce propos, la situation d'Epstein ne semble pas avoir été facile : il ne fait pas partie du cercle des traducteurs professionnels ayant une formation «classique ». S'il se montre souvent très critique vis-à-vis de leur travail, on peut se demander quelle est la part de son opportunisme quand il dénigre, auprès de Schnitzler, leur action. Voici deux exemples :

20. - D'après Epstein, ce serait une allusion à Jean-François Collin d'Harleville (1755-1806), membre de l'Académie Française et auteur de comédies qui a connu un certain succès XVIIII siècle. 
Dans une lettre du 15 mai 1902, alors qu'il est en train de traduire Der grüne Kakadu, il prétend que c'est la traduction de Maurice Vaucaire qui aurait causé la disparition rapide de La Compagne du répertoire du Théâtre Antoine ${ }^{21}$ :

Ich persönlich halte Vaucaire fuer einen vorzüglichen VaudevilleUebersetzer; fuer litterarische Dinge ist aber die Kenntniss [sic] der deutschen Sprache meiner Ueberzeugung nach unerlaesslich und mit dieser Unkenntniss [sic] hat auch Thorel an allen Enden zu kaempfen. Nur aus diesen Umstaenden laesst sich erklaeren, dass die « Genossin » so absolut ins Wasser gefallen ist.

(je tiens Vaucaire pour un excellent traducteur de vaudevilles; mais pour des textes plus littéraires, la connaissance de la langue allemande est indispensable; à Thorel aussi d'ailleurs la méconnaissance [de cette langue] cause des problèmes. Ce sont seulement ces circonstances qui peuvent expliquer pourquoi la «Compagne » a été un tel four).

Le 23 décembre 1903, c'est sur Maurice Rémon que tombent les foudres d'Epstein. À propos d'une éventuelle traduction et mise scène de Les Derniers masques (du cycle Heures vives), il qualifie Maurice Rémon de "Schullehrer an einem Collège » et rapporte les propos d'Antoine : «C'est un pompier et non un dramaturgiste [sic] »; quant à Noémi Valentin, son apport à la traduction serait négligeable (« Die Valentin zählt überhaupt nicht »). Schnitzler, qui ne veut pas compromettre, par une traduction inadéquate, la possibilité d'une mise en scène de sa pièce, propose donc à Epstein de prendre contact avec Rémon et de retravailler le texte ${ }^{22}$.

Les propos d'Epstein contiennent sans doute une part de vérité. Il faut cependant avoir à l'esprit qu'il essaie de se placer auprès de Schnitzler comme agent littéraire exclusif en France. Souvent, dans la correspondance, Epstein laisse entendre que ses relations avec les autres traducteurs sont tendues : "Rémond [sic] et Thorel voient ma position d'un mauvais œil et, quand je vais les voir, ils me jettent dehors ». Il leur reproche avant tout de n'avoir aucune connaissance du théâtre allemand et d'avoir proposé des pièces à Antoine qui ne valent pas la peine d'être

21. - La mise en scène de cette pièce en un acte au Théâtre Antoine n'a effectivement connu que quatre représentations entre le 29 avril et le 4 mai 1902. La traduction de Maurice Vaucaire a été publiée dans la Revue bleue du 24 mai 1902 (4e série, XVII, $\left.\mathrm{n}^{\circ} 21\right)$.

22. - Cette proposition ne semble pas avoir été suivie tout de suite du résultat escompté. L'acte Les Derniers masques ne sera monté que huit ans plus tard, en avril 1912. Si le spectacle a bien pour cadre le Théâtre Antoine, c'est dans une production du Théâtre de L'Euvre et dans une mise en scène d'Aurélien Lugné-Poe, la traduction étant signée Maurice Rémon. Le texte paraît dans la Revue bleue en deux livraisons les 16 et 23 novembre 1912. 
montées. Les exemples que donne Epstein semblent lui donner raison, car il mentionne deux pièces complètement oubliées de nos jours : Das schwarze Schäflein, une pièce en cinq actes de Richard Skowronnek (1862-1952) parue à Berlin en 1902, et Alt-Heidelberg.Le Théâtre Antoine crée effectivement le 29 janvier 1906 Le Vieil Heidelberg, une pièce en cinq actes de Wilhelm Meyer-Förster (1862-1934) dans une traduction de Maurice Rémon et Wilhelm Bauer. Cette pièce sentimentale, qui est une adaptation, par l'auteur, de son propre roman Karl Heinrich (1899), rassemble bon nombre de clichés sur les rapports «idylliques » entre les régnants et le peuple dans les royaumes et les principautés allemands. Elle semble avoir connu - étonnamment ? - un certain succès auprès du public d'Antoine : le registre du Théâtre Antoine fait effectivement état de 69 représentations entre janvier et mai 1906.

Les relations entre Epstein et Schnitzler se détériorent au cours de l'année 1907. Le 29 septembre 1908 l'écrivain viennois écrit à Epstein :

[...] entschlossen, die Vertretung meiner Angelegenheiten in Paris anderweitig zu vergeben, danke Ihnen heute für Ihre bisher aufgebrachte Mühe und bitte Sie, mir recht bald die Vollmacht zurückzusenden, die ich Ihnen vor einigen Jahren geschickt habe.

Epstein s'exécutera, mettant de ce fait un terme à une relation largement problématique qui ne cessait de se dégrader. Tout aussi largement problématiques apparaissent les conditions même dans lesquelles des agents du transfert culturel sont amenés à faire office, dans l'appréciation que l'on en a après-coup, d'intermédiaires et de passeurs. Le concours hautement précieux qu'ils apportent dans la circulation des œuvres et des idées se révèle en effet tout autant le fruit du hasard que celui d'une ferme résolution; il traduit, comme le montrent les exemples sur lesquels nous nous sommes appuyé, aussi bien le choix de satisfaire à des impératifs économiques que de se mettre au service d'une œuvre ou d'un auteur dans lesquels on croit. 